\title{
Wie Sojaisoflavone vor Erdnussallergie schützen ...
}

\section{Obwohl die Proteine von Erdnüssen und Sojabohnen starke Homo- logien aufweisen, sind allergische Reaktionen gegen Soja seltener und weniger gravierend. Möglicherweise können die in Soja enthaltenen Isoflavone die Immunreaktion auf Erdnussallergene unterdrücken.}

Fo dies orscher aus New York, USA, haben diese Hypothese im Tiermodell und in vitro überprüft. Dazu ernährten sie Mäuse entweder mit einem sojafreien Futter oder mit einem Futter, dem die

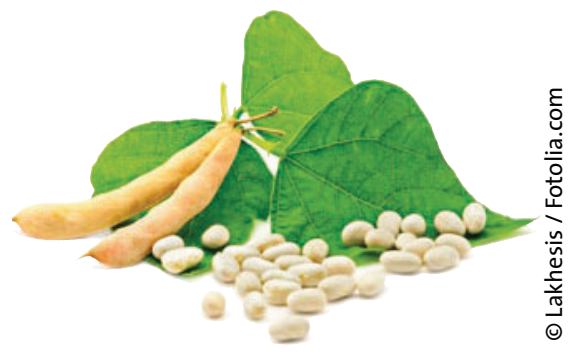

Die Sojabohne ist reich an Isoflavonen, die im Mausmodell für Erdnussallergie die Reifung von dendritischen Zellen und die $\mathrm{CD}_{4}^{+}$-Zell-Antwort unterdrücken.
Sojaisoflavone Genistein und Daidzein zugesetzt waren. Die Tiere wurden dann gegen Erdnüsse sensibilisiert und schließlich einem Provokationstest unterzogen.

Bei den Mäusen mit isoflavonhaltiger Diät traten signifikant weniger anaphylaktische Symptome auf. Dementsprechend ließen sich bei ihnen nach der Provokation auch weniger degranulierte Mastzellen in der Haut nachweisen als bei sojafrei ernährten Mäusen. Die Serumspiegel von erdnussspezifischen $\mathrm{IgE}$ und IgG2a-Antikörpern waren ebenfalls deutlich reduziert. Durch die Ernährung mit Isoflavonen wurde außerdem die durch Choleratoxin induzierbare Reifung von dendritischen Zellen inhibiert.

Der Effekt auf die dendritischen Zellen (DC) war auch bei humanen DC in Kultur nachvollziehbar: Eine Stimulation mit Choleratoxin bewirkte in Gegenwart von Sojaisoflavonen eine schwächere Hochregulation der Markerproteine CD83 und CD80 als ohne Isoflavone. Durch Genistein wurde außerdem die Zytokinfreisetzung aus den aktivierten DC reduziert. Die durch DC vermittelte Aktivierung von $\mathrm{CD}^{+}$-Zellen war in Anwesenheit des Isoflavons ebenfalls vermindert.

Fazit: In einem Mausmodell für erdnussinduzierte Anaphylaxie schützen Sojaisoflavone vor der allergischen Sensibilisierung. Dieser Effekt beruht auf einer Immunmodulation, bei der die Reifung von dendritischen Zellen und die $\mathrm{CD}^{+}{ }^{+}$ Zell-Antwort unterdrückt werden. Die Supplementierung mit Sojaisoflavonen könnte daher eine neue Strategie darstellen, um Erdnussallergien zu verhindern.

Dr. Beate Schumacher

Masilamani $\mathbf{M}$ et al. Soybean isoflavones regulate dendritic cell function and suppress allergic sensitization to peanut. J Allergy Clin Immunol 2011 Jun 22; DOI:10.1016/j.jaci.2011.05.009

\section{... und Keimexposition von Schwangeren auf den Nachwuchs wirkt}

\section{Asthmaprävention beginnt schon im Mutterleib. Protektiv wirkt z.B. der mütterliche Kontakt mit bestimmten Keimen. Er kann offenbar epigenetische Veränderungen anstoßen, welche die Immunantwort modulieren. Zu diesem Ergebnis führen Untersuchungen an einem Mausmodell, die an der Universität Marburg durchgeführt wurden.}

\footnotetext{
rächtigen Mäusen wurde intranasal das apathogene gramnegative Bakterium Acinetobacter (A.) lwoffii F78 oder reine Pufferlösung appliziert. Die Nachkommen von keimexponierten Müttern entwickelten nach Sensibilisierung und Provokation mit Ovalbumin signifikant weniger klinische Zeichen eines Asthmas. Gleichzeitig kam es zu einer deutlichen Verschiebung im TH1-/TH2-Gleichgewicht: Mononukleäre Zellen der Milz produzierten mehr $\gamma$-Interferon (IFN- $\gamma$ ) und weniger von den TH2-Zytokinen Interleukin(IL)-4, IL-5 und IL-13.
}

Der durch $A$. lwoffii F78 induzierte Asthmaschutz war IFN- $\gamma$-abhängig: Eine Behandlung des Nachwuchses mit neutralisierenden Antikörpern gegen IFN- $\gamma$ resultierte in einer Entzündung und Hyperreagibilität der Atemwege und einer erhöhten Produktion von TH2Zytokinen.

Die vermehrte IFN- $\gamma$-Synthese nach mikrobieller Exposition der Mutter wird wahrscheinlich durch epigenetische Prozesse vermittelt. So war am IFN- $\gamma$-Promotor in $\mathrm{CD}^{+}{ }^{+}-\mathrm{CD} 25^{-}$-Zellen eine stärkere Histon-H4-Acetylierung feststellbar als in Zellen von Kontrolltieren. Eine pharmakologische Blockade der H4Acetylierung verhinderte die Ausbildung des asthmaprotektiven Phänotyps.

Fazit: In einem Mausmodell verändert mütterlicher Keimkontakt die Histonacetylierung des IFN- $\gamma$-Promotors in der nächsten Generation. Dadurch verstärkt sich die IFN- $\gamma$-Synthese, wodurch TH2-abhängige allergische Reaktionen und die Ausbildung eines Asthmas verhindert werden. Diese Studienergebnisse stützen die Hygienehypothese und sie zeigen, wie Gene und Umwelt beim Schutz vor Allergien über epigenetische Prozesse zusammenwirken können. Dr. Beate Schumacher

Brand S et al. Epigenetic regulation in murine offspring as a novel mechanism for transmaternal asthma protection induced by microbes. J Allergy Clin Immunol 2011 Jun 22; DOI:10.1016/j.jaci.2011.04.035 